\title{
MODELO PARA LA RAMA DESCENDENTE DE ESPECTROS DE DISEÑO SÍSMICO Y APLICACIONES AL CASO DE LA CIUDAD DE MEDELLÍN
}

\author{
Juan Diego Jaramillo ${ }^{(1)}$
}

\begin{abstract}
RESUMEN
Se propone en este artículo una forma para la rama descendente de espectros para diseño sísmico en la superficie del suelo. La forma propuesta considera para su formulación el modelo $w^{2}$ para el espectro de amplitudes de Fourier a nivel del basamento rocoso y la función de transferencia del suelo, y a partir de estos y la teoría de vibraciones aleatorias, $T V A$, la construcción de espectros de respuesta de aceleraciones a nivel del basamento rocoso, funciones de amplificación de espectros de respuesta, y finalmente, espectros de respuesta de aceleraciones a nivel de la superficie del suelo. Para efectos de comparación, se estiman con esta propuesta los espectros de aceleración y desplazamientos que resultarían para la microzonificación sísmica de Medellín (U. EAFIT, et al., 1999) y se comparan con los que rigen actualmente y con los presentados por Jaramillo (2002) producto del procesamiento y ajuste de los acelerogramas registrados por la Red acelerográfica de Medellín, RAM.
\end{abstract}

\section{SUMMARY}

A form for descendent zone of seismic design spectrum in the surface of the ground is proposed in this article. The proposed form considers for its formulation the soil transfer function and the $w^{2}$ model for the Fourier amplitude spectrum at the rock basement level, and from these and using the random vibrations theory, TVA, construction of acceleration response spectrum at rock basement level, amplification functions of response spectrum, and finally, acceleration response spectrum at the surface of the ground, are made. For comparison effects, the acceleration response spectrum considered with this proposal and displacements response spectrum for the seismic microzonification of Medellín (U. EAFIT et al., 1999) are calculated and compared with those which prevail at the moment and with those presented by Jaramillo (2002) product of the processing and fits of accelerograms registered by the Medellín accelerographic Network, RAM.

\section{INTRODUCCIÓN}

Muchas de las reglamentaciones para diseño sísmico alrededor del mundo especifican el sismo para diseño a través de un espectro de diseño de seudoaceleraciones. En el caso más general este espectro, por simplicidad en su formulación, se ha dividido en tres partes: la zona de periodos bajos, definida a través de una recta que parte de la aceleración máxima esperada en la superficie

Artículo recibido el 10 de abril de 2002 y aprobado para su publicación el 7 de febrero de 2003. Se aceptarán comentarios y/o discusiones hasta cinco meses después de su publicación.

${ }^{(1)}$ Departamento de Ingeniería Civil, Universidad EAFIT, A.A. 3300, Medellín, Colombia, jiarami@eafit.edu.co 
del suelo y que termina en la aceleración espectral máxima; la zona de periodos intermedios o periodos de amplificación máxima, definida por una constante que representa la aceleración espectral máxima especificada, y la tercera rama, o rama descendente, tradicionalmente se ha especificado proporcional a $1 / T^{\alpha}$.

De acuerdo con las formas descritas, el espectro de diseño queda completamente definido en términos de cinco parámetros: la aceleración máxima a nivel de la superficie del suelo, $A_{\max }$, la aceleración espectral máxima, $S a_{\max }$, el primer periodo de quiebre del espectro, $T_{1}$, que define la zona de periodos bajos, el segundo periodo de quiebre del espectro, $T_{2}$, que define el final de la meseta de amplificación máxima, y el exponente $\alpha$, que define la forma de la caída del espectro. En la fig. 1 se muestra esta forma espectral y los parámetros que la definen.

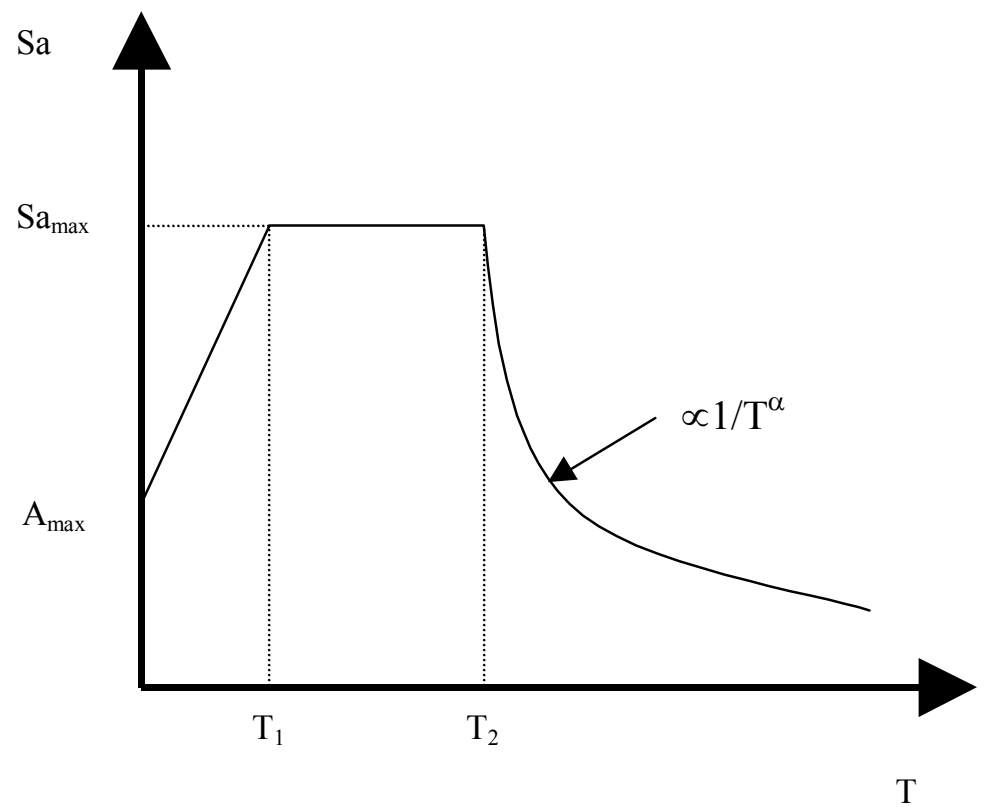

Figura 1. Forma del espectro de aceleraciones para diseño

El argumento que ha impuesto la definición del parámetro $\alpha$, que controla la caída del espectro, parece estar asociado a la mayor seguridad que se le debe dar a las estructuras de periodos altos, que generalmente representan las grandes obras de la ingeniería como son los edificios altos, puentes, etc. Igualmente, cuando se propone $\alpha=1.0$, parece responder al hecho de que el espectro a nivel del basamento rocoso a continuación de la zona de aceleración constante, presenta una zona de seudovelocidad constante. Esta observación, sustentada en la forma sugerida por el modelo $w^{2}$ para el espectro de amplitudes de Fourier en el basamento rocoso, y modificada por la función de transferencia del suelo, son la base de la propuesta que aquí se presenta y que se detalla más adelante.

En el Código Colombiano de Construcciones Sismorresistentes de 1984 (AIS, 1984) se propuso para $\alpha$ un valor de 2/3, que se cambió en la versión de 1998 de esta reglamentación a un valor de 1.0. Las últimas normas americanas, NEHRP (1997), UBC (1997) e IBC (2000), proponen para $\alpha$ un valor de 1.0, independientemente de si se trata del espectro de diseño en el 
basamento rocoso o en la superficie del suelo (Leyendecker et al., 2000; Dobry et al. 2000). La tendencia señalada por estas últimas normas se ha difundido en los países con alta y mediana amenaza sísmica en América Latina, como es el caso ya señalado de Colombia, de Venezuela (ACFMN, 1997) y El Salvador, (ASIA, 1997). Argentina (SIREA, 1991) mantiene una caída del espectro de diseño tanto para el basamento rocoso como para suelos blandos definida por un exponente $\alpha=2 / 3$.

El Código Nacional de Construcciones del Canadá (ACNBC, 1995) no es diferente a lo establecido en las normas de los Estados Unidos; independientemente de si se trata del espectro de diseño en roca o en la superficie del suelo, estos caen inversamente proporcional al periodo, es decir, $\alpha=1.0$.

Hasta el momento y a juicio del autor, a nivel de América la única región que ha propuesto algo diferente para la rama descendente de los espectros para diseño sísmico es la ciudad de México, que en la actual propuesta de las Normas Técnicas Complementarias para Diseño por Sismo (NTCS-2001, 2001), propone para la zona I o de terreno firme un coeficiente $\alpha=1.0$, en la zona II o de transición un coeficiente $\alpha=1.33$, y finalmente en la zona III o de depósitos blandos, un coeficiente $\alpha=2.0$, indicando que a medida que el suelo se va haciendo más blando el coeficiente de caída del espectro de diseño es más alto, representando caídas más pronunciadas. El trabajo de Ordaz et al. (2000) es la base para estas modificaciones a los espectros para diseño de la ciudad de México.

Para terminar la revisión de códigos actuales, el Eurocódigo (IAEE, 1996), independientemente de si se trata del espectro para diseño en roca o en la superficie de suelos blandos, propone dos ramas descendentes en los espectros de diseño: Una primera etapa a continuación de la zona de aceleración constante y hasta $3.0 \mathrm{~s}$, con una caída definida por un exponente $\alpha=1.0$, representando la zona de seudovelocidad constante. A partir de este periodo se define una caída más pronunciada con $\alpha=2.0$, que limita el crecimiento del espectro de desplazamiento.

Es claro de la revisión de los códigos que para diseño sísmico se utilizan actualmente en algunos países, exceptuando la actual propuesta de las Normas Técnicas Complementarias para Diseño por Sismo en la ciudad de México, que el hecho de que la respuesta sea medida en roca o en suelo blando, no modifica la forma de caída de los espectros de diseño, es decir, que los efectos locales solo modifican en algunos casos las aceleraciones espectrales máximas, $S a_{\max }$, y en otros códigos, adicionalmente a lo anterior, los periodos de quiebre del espectro $T_{1}$ y $T_{2}$ (fig. 1). Esta tendencia es a todas luces contraria al hecho de que los espectros de Fourier de las señales registradas en suelos blandos se pueden estimar como el producto de los espectros de Fourier de los registros en el basamento rocoso, multiplicados por la función de transferencia del suelo, que como se verá más adelante, modifica de manera apreciable las formas de caída de los espectros de respuesta a nivel de la superficie de suelos blandos.

Las caídas de los espectros de respuesta de sismos registrados en suelos blandos son en todos los casos mucho más pronunciadas que lo que hasta ahora se ha propuesto en gran parte de los reglamentos sísmicos. No se ve, entonces, ningún otro argumento diferente al de la seguridad 
de estructuras de periodos altos, mencionado antes, que justifique los valores hasta ahora propuestos para el parámetro $\alpha$ que define la caída de los espectros de diseño en suelos blandos.

El interés repentino por tratar de encontrar un mejor ajuste a las formas de caída de los espectros de diseño en suelos blandos no es caprichoso: obedece a razones de más fondo que la simple meticulosidad en la forma espectral propuesta para diseño. En la mayoría de los códigos sísmicos alrededor del mundo se ha introducido el control de derivas como componente fundamental en el diseño sismo-resistente de edificaciones, obligando al cálculo de los desplazamientos de la estructura. Este cálculo no es novedoso en sí, es el resultado, que juega un papel importante en el diseño de la estructura. Las versiones más recientes de algunos códigos para diseño sísmico (NSR-98; NEHRP,1997; IBC, 2000), tratando de controlar más los daños a elementos no estructurales adheridos a la estructura, volvió más estricto el control de las derivas, reduciendo sustancialmente el límite permitido para éstas. Los calculistas coinciden en que actualmente, para la mayoría de las estructuras, el control de derivas es el estado límite que define la geometría y disposición de los elementos estructurales dentro de la edificación. De acuerdo con lo anterior, resulta que para el diseño como lo conciben hoy muchos reglamentos para diseño sísmico alrededor del mundo, es fundamental una estimación muy precisa de los desplazamientos que tendría la estructura cuando esta se vea sometida a los sismos de diseño y control de daños.

En la fig. 2 se muestran los desplazamientos máximos que tendría un sistema de un grado de libertad cuando la respuesta estructural está especificada en términos del espectro de respuesta de seudoaceleraciones (espectros de diseño) propuestos en algunas versiones actuales de normas para diseño sísmico. En otras palabras, se muestran los espectros de desplazamiento con base en los espectros de diseño. Con trazo continuo se incluye un espectro de desplazamiento que sigue los principios de la dinámica estructural: el desplazamiento espectral es asintótico al desplazamiento máximo del terreno a medida que aumenta el periodo estructural.
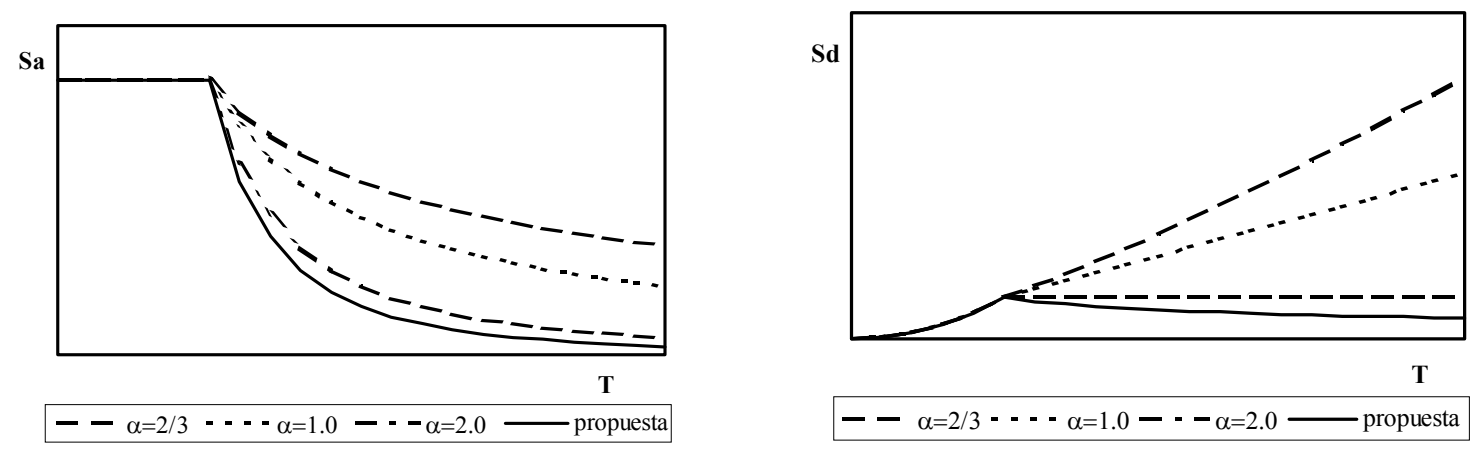

Figura 2. Espectros de diseño propuestos en algunos códigos para diseño sísmico y los espectros de desplazamiento asociados

Es claro de la fig. 2 que a partir del periodo de quiebre $T_{2}$ muchas reglamentaciones actuales sobrestiman sistemáticamente y de manera creciente con el periodo estructural, el desplazamiento máximo esperado. Más grave todavía es que algunas de las normativas en 
discusión, violan los principios de la dinámica estructural cuando postulan desplazamientos máximos del terreno infinitos.

Esta discrepancia notable es evidente en toda su dimensión ahora que el cálculo de los desplazamientos es crucial en el diseño sismo-resistente de edificaciones debido a los estados límite que establecen las normas. Si el diseño estuviese enteramente controlado por la resistencia, dictada por el espectro de seudoaceleraciones, el impacto sería mucho menor debido a que las aceleraciones de diseño decrecen con el periodo, aunque se estarían igualmente sobreestimando sistemáticamente éstas en la zona descendente del espectro.

En lo que sigue y con base esta vez en modelos físicos del espectro de amplitudes de Fourier en el basamento rocoso y en la función de transferencia del suelo, y haciendo uso de la teoría de vibraciones aleatorias, $T V A$, se propone una forma funcional para la rama descendente del espectro en términos del periodo en el que se presenta la amplitud máxima en la roca, $T r$, del periodo fundamental del depósito de suelo, $T_{s}$, del amortiguamiento del suelo, $\xi$, y de la impedancia entre el suelo y la roca, $I$.

\section{ESPECTROS DE DISEÑO VS ESPECTROS DE RESPUESTA}

A continuación se definirá lo que se entiende por espectros de diseño y de respuesta y se aclarará la relación que hay entre ellos para justificar el procedimiento que se propone para estimar los primeros con base en procedimientos claramente establecidos para estimar los segundos.

Por definición, los espectros de aceleraciones para diseño no representan el espectro de respuesta de aceleraciones de una serie de tiempo que pueda considerarse como el acelerograma de diseño; más bien se parece más, o se fundamenta en lo que se ha llamado espectro de amenaza uniforme, que se define como el trazo que une las aceleraciones espectrales asociadas independientemente a cada periodo estructural con una probabilidad de excedencia definida, en un periodo de tiempo también definido. En otras palabras, el espectro de amenaza uniforme es el trazo que une las aceleraciones espectrales asociadas al mismo periodo de retorno, trabajando cada periodo estructural independientemente. Este espectro de amenaza uniforme se modifica un poco para simplificar sus trazos y para considerar en algunos casos incertidumbres provenientes del cálculo del periodo estructural de la edificación.

De la definición de espectros de diseño se desprende que, como un todo, no mirando cada periodo estructural independientemente, la energía total involucrada bajo ese trazo supera la energía total, como medida de la intensidad del sismo que se calcularía suponiendo la misma probabilidad de excedencia y en el mismo periodo de tiempo usados para el cálculo del espectro de amenaza uniforme. En otras palabras, el espectro de amenaza uniforme se puede ver como la envolvente de los espectros producidos por los sismos "máximos creíbles" de cada una de las sismofuentes que afectan el sitio en estudio. Esta última definición debe tomarse únicamente como ilustrativa, pues rigurosamente no es claro ni el procedimiento para efectuar el cálculo descrito ni la definición de "sismo máximo creíble". 
Si cada edificación se pudiera asociar estrictamente a un sistema de un solo grado de libertad, no como la combinación de varios de ellos, la sobre-estimación descrita en los espectros que para diseño estamos utilizando no tendría ninguna incidencia, y es sobre este supuesto que se basa la metodología descrita para la obtención de los espectros de diseño. En general, aunque cualquier sistema se representa como la combinación de varios sistemas de un solo grado de libertad, uno de ellos, llamado el fundamental, participa en una proporción mucho mayor que los demás en la respuesta final del sistema. Es claro que mientras más alta sea la participación del modo fundamental, más justificable es el procedimiento descrito para la obtención del espectro de diseño. En los casos en que esta premisa no se cumpla, se obtiene un sobre diseño que, hasta donde se sabe, es sano, dado que estos casos normalmente ocurren en edificaciones con asimetrías en planta o elevación, que generan concentraciones de esfuerzos y otras medidas de la respuesta estructural en algunas zonas, y que no están completamente previstas en las metodologías que para diseño se usan.

Aunque ya se mencionó que los espectros para diseño no son exactamente iguales a los espectros de respuesta de acelerogramas de diseño, las formas espectrales calculadas de acelerogramas registrados o propuestos con base en modelos físicos se asemejan a las formas espectrales de diseño, con trazos más suaves y envolventes estos últimos, que permiten decidir sobre los rangos de valores de algunos de los parámetros que definen los espectros de diseño (Chopra, 2001). Por lo anterior, es sensato calcular $\alpha$ ajustando los espectros de diseño a formas espectrales propuestas de espectros de respuesta calculados de sismos registrados o de modelos propuestos.

\section{PROPUESTA}

El modelo $w^{2}$, ver por ejemplo Boore, 1983, sugiere la siguiente forma funcional para el espectro de amplitudes de Fourier de la aceleración en el basamento rocoso:

$$
A(f) \propto \frac{f^{2}}{1+\left(\frac{f}{f_{c}}\right)^{2}} \frac{1}{1+\left(\frac{f}{f_{m}}\right)^{2 s}} \frac{e^{-\pi f R / \mu Q}}{R}
$$

donde $f_{c}$ es la llamada frecuencia de esquina, relacionada con el momento sísmico, $M_{o s}$, a través de la siguiente ecuación, Brune, 1970.

$$
f_{c}=4.9 \times 10^{6} \mu\left(\Delta \sigma / M_{o s}\right)^{1 / 3}
$$

donde $\mu$ es la velocidad de propagación de las ondas $S$ en $\mathrm{km} / \mathrm{s}, Q$ es el llamado factor de calidad que controla la atenuación anelástica, $\Delta \sigma$ mide la llamada caída de esfuerzos en bares, y los parámetros $f_{m}$ y $s$ controlan el decaimiento en alta frecuencia. 
El momento sísmico, $M_{o s}$ en ergios, está relacionado con la magnitud $M_{w}$ a través de la expresión (Hanks y Kanamori, 1977):

$\log M_{o s}=1.5 M_{w}+16.1$

El modelo $w^{2}$ fue deducido considerando una fuente puntual y solo el efecto de las ondas de corte $S$, lo que impone las siguientes dos limitaciones al modelo y a los resultados del presente trabajo. Para fuentes más lejanas a aproximadamente $100 \mathrm{~km}$ el movimiento deja de estar controlado por las ondas $S$, por lo que deja de ser aplicable. Igualmente, para puntos de observación muy cercanos al área de ruptura se presentan fenómenos de saturación de la aceleración que deben considerarse. A este respecto, Singh et al. (1989) propusieron un modelo sismológico que considera el tamaño finito de la fuente y que refleja el fenómeno de saturación de la aceleración mencionado. Modelos como este deberían ser utilizados en casos de fuentes muy cercanas, menores a cinco veces el radio de la falla circular equivalente de ruptura.

La ec. 1 sugiere un espectro de amplitudes de Fourier que crece con el cuadrado de la frecuencia hasta la frecuencia de esquina $f_{c}$. Después de esta frecuencia el espectro es aproximadamente plano hasta la frecuencia $f_{m}$, en donde se inicia la caída de amplitudes en la alta frecuencia.

Si se considera, como es usual, que $f_{m}$ tiene valores superiores a los $3 \mathrm{~Hz}$, esto es, periodos inferiores o iguales a $0.33 \mathrm{~s}$, se concluye que la rama descendente de los espectros de respuesta en función del periodo y a nivel del basamento rocoso está controlada, en la parte inicial por la zona plana del espectro de amplitudes de Fourier, y en la zona final de periodos altos, $T_{c}$, mayores o iguales a $1 / f_{c}$, por la rama ascendente en frecuencia y proporcional a $f^{2}$.

En la tabla 1 se muestran, con base en las ecs. 2 y 3 , los periodos $T_{c}$ en que el espectro de respuesta a nivel del basamento rocoso comienza a comportarse en función de la rama ascendente en frecuencia. En todos los casos se considera una velocidad de propagación de las ondas $S$ igual a $3.2 \mathrm{~km} / \mathrm{s}$.

Tabla 1. Periodos $T_{c}=1 / f_{c}$ para diferentes magnitudes $M_{w}$ y dos caídas de esfuerzo $\Delta \sigma$.

\begin{tabular}{|c|c|c|}
\hline \multirow{2}{*}{$M_{w}\left(\rho^{\circ}\right.$} & \multicolumn{2}{|c|}{$T_{c}(s)$} \\
\cline { 2 - 3 } & $\Delta \sigma=100$ bares & $\Delta \sigma=200$ bares \\
\hline 5.0 & 1.0 & 0.8 \\
\hline 5.5 & 1.8 & 1.4 \\
\hline 6.0 & 3.2 & 2.5 \\
\hline 6.5 & 5.7 & 4.5 \\
\hline 7.0 & 10.1 & 8.0 \\
\hline 7.5 & 18.0 & 14.3 \\
\hline 8.0 & 32.0 & 25.4 \\
\hline
\end{tabular}


De la Tabla 1 es claro que para magnitudes de momento superiores a $6.5^{\circ}$, la rama descendente del espectro de respuesta hasta $4.0 \mathrm{~s}$ está controlada por la parte plana del espectro de amplitudes de Fourier.

A continuación, y haciendo uso de la teoría de vibraciones aleatorias, Cartwright y Longuett-Higgins (1956), que permite estimar la esperanza del valor máximo de una serie de tiempo si de esta solo se conoce su espectro de amplitudes de Fourier y la duración de la fase intensa, se calculan los espectros de respuesta a nivel del basamento rocoso asociados a espectros de amplitudes de Fourier como los descritos por la ec. 1, para múltiples valores de los parámetros $f_{c}, f_{m}, s, Q, \mu$ y $R$ que lo definen.

Para la duración de la fase intensa del movimiento en el basamento rocoso, $T_{d}$, se consideró la siguiente expresión (Herman, 1985):

$$
T_{d}=\frac{1}{f_{c}}+0.05 R
$$

que modificada por los parámetros del oscilador, $\xi$ y $f_{o}$, resulta en la siguiente duración para la fase intensa del movimiento de este, $T_{o}$, (Boore y Joyner, 1984):

$$
T_{o}=T_{d}+\frac{u^{3}}{2 \cdot \pi \cdot \xi \cdot f_{o}\left(u^{3}+\frac{1}{3}\right)}
$$

donde $u=f_{o} \cdot T_{d}$

Resultado de este proceso, como se esperaba, es que la forma del espectro de respuesta de aceleraciones a nivel del basamento rocoso decae aproximadamente de manera inversa al periodo para periodos mayores a $1 / f_{m}$ y menores a $1 / f_{c}$. Lo anterior confirma que entre los periodos mencionados se está en la zona de seudovelocidad constante.

Es importante destacar también que para la mayoría de las aplicaciones los espectros de diseño están asociados a eventos con magnitudes de momento iguales o superiores a $6.5^{\circ}$, en cuyo caso, el periodo hasta donde se alargaría la zona de seudovelocidad constante estaría más allá de $\operatorname{los} 4.0 \mathrm{~s}$.

Igualmente, es posible concluir de los espectros de respuesta estimados, que el periodo en el que el espectro de respuesta alcanza la máxima amplitud es aproximadamente igual a $1 / f_{m}=T_{r}$, y que esta amplitud, relativa a la aceleración máxima a nivel del basamento rocoso, $A_{a}$, se puede estimar con muy buena aproximación con la siguiente expresión:

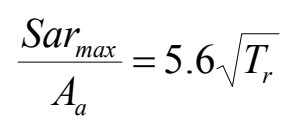


Que expresa, por ejemplo, que cuando $T_{r}=0.2 \mathrm{~s}$, la amplificación espectral máxima a nivel del basamento rocoso relativa a la aceleración máxima en la roca, es igual a 2.5, que ha caracterizado por muchos años los espectros de diseño sísmico a nivel de roca. Para casos de sismos lejanos en donde la alta frecuencia se filtra casi completamente, por ejemplo $T_{r}=0.4 \mathrm{~s}$, el factor de amplificación sobre la aceleración máxima en roca para alcanzar la aceleración espectral máxima resulta igual a 3.5 .

Los efectos locales debidos al depósito de suelo en el sitio se consideran a través de la función de transferencia entre las aceleraciones absolutas en el basamento rocoso semi-infinito medidas a nivel de la superficie (outcropping), y las aceleraciones absolutas medidas en la superficie del depósito de suelo, que se escribe (Newmark y Rosenblueth, 1971):

$$
F T=\frac{1}{\cos \left(\frac{\pi}{2} \beta\right)+\frac{i}{I} \operatorname{sen}\left(\frac{\pi}{2} \beta\right)}
$$

donde $i=\sqrt{-1}, \beta$ es la relación de frecuencias de excitación, $f$, y la frecuencia natural del depósito de suelo, $f_{s}$,

$$
\beta=\frac{\bar{f}}{f_{s}}
$$

e $I$ es la impedancia en el contacto entre el basamento rocoso y el depósito de suelo, definida como:

$$
I=\frac{\rho_{r} C_{r}}{\rho_{s} C_{s}}
$$

donde:
$\rho_{r} \quad$ Densidad de la roca
$\rho_{s} \quad$ Densidad del suelo
$C_{r} \quad$ Velocidad de la onda de corte en la roca
$C_{s} \quad$ Velocidad de la onda de corte en el suelo

Esta función de transferencia (ec. 7) es aplicable únicamente al caso en que se consideran nulos los amortiguamientos del basamento rocoso y del depósito de suelo. Para el caso en el que se considera un amortiguamiento de tipo histerético en el depósito de suelo, la función de transferencia se escribe (Avilés et al., 1995):

$$
F T=\frac{1}{\cos \left(\frac{\pi}{2} \beta\left(1-i \xi_{s}\right)\right)+\frac{i}{I} \operatorname{sen}\left(\frac{\pi}{2} \beta\left(1-i \xi_{s}\right)\right)}
$$


donde $\xi_{s}$ es la razón de amortiguamiento del suelo respecto al crítico.

Si se multiplica el espectro de amplitudes de Fourier del movimiento en el basamento rocoso sugerido por el modelo $w^{2}$ y definido por la ec. 1, por la amplitud de la función de transferencia definida por la ec. 10, se obtiene el espectro de amplitudes de Fourier de la aceleración absoluta en la superficie del suelo. Ahora, con la ayuda de la teoría de vibraciones aleatorias, es posible, igual que se hizo para el movimiento en el basamento rocoso, estimar el espectro de respuesta de aceleraciones en la superficie del suelo.

En este caso, la duración de la fase intensa en la base de la estructura, $T_{d}$, no debe ser la duración de la excitación en el basamento rocoso sino la duración de la respuesta del depósito de suelo, $T_{d s}$, que, nuevamente, puede obtenerse con la expresión debida a Boore y Joyner (1984):

$$
T_{d s}=T_{d}+\frac{u^{3}}{2 \cdot \pi \cdot \xi_{s} \cdot f_{s}\left(u^{3}+\frac{1}{3}\right)}
$$

donde $u=f_{s} \cdot T_{d}$

Esta duración de la fase intensa del movimiento en la superficie del suelo se modifica nuevamente para considerar la duración de la fase intensa del movimiento del oscilador de acuerdo a la misma expresión, pero esta vez considerando los parámetros del oscilador, $\xi$ y $f_{o}$.

Finalmente, se calcula la función de amplificación de espectros de respuesta, $R R S$, por sus siglas en inglés, como el cociente entre los espectros de respuesta de aceleración en la superficie del suelo y en el basamento rocoso.

La caracterización de la función $R R S$ es importante porque, como se mencionó en la introducción, los espectros de amenaza uniforme sobre los que se basan los espectros para diseño sísmico están fundamentados en el concepto de espectros de respuesta, así que si se tiene el espectro de amenaza uniforme en roca, basta con multiplicarlo por la función RRS asociada al suelo en el sitio para obtener el correspondiente espectro de amenaza uniforme en la superficie del suelo.

La afirmación anterior implica dos simplificaciones que se justifican a continuación. En primer lugar, el espectro de amenaza uniforme en la superficie del suelo se obtiene del producto del espectro de amenaza uniforme en la roca por la función $R R S$ solo si no hay incertidumbre en esta última función, es decir, se conoce con absoluta certeza. Si hay incertidumbre, esta puede considerarse de manera simplificada definiendo una función $R R S$ equivalente, $R R S_{e q}$, como:

$$
R R S_{e q}=\overline{R R S}+n \sigma_{R R S}
$$

donde $\overline{R R S}$ es la media de $R R S$ y $\sigma_{R R S}$ su desviación. 
En lo que sigue se considera que la función de amplificación, $R R S$, se puede expresar como:

$R R S=\overline{R R S} \cdot \varepsilon$

donde $\varepsilon$ es una variable aleatoria de distribución lognormal con esperanza igual a 1.0 y desviación igual a $\sigma_{\varepsilon}$. Esta última relación pone de presente el hecho de que la incertidumbre sobre la función de amplificación aumenta a medida que aumenta el valor medio de la amplificación, tendencia sugerida por los datos experimentales.

De acuerdo con lo anterior, la función $R R S_{\text {eq }}$ se puede expresar como:

$$
R R S_{e q}=\overline{R R S}\left(1.0+n \sigma_{\varepsilon}\right)
$$

Como resultado de la microzonificación sísmica de cinco ciudades en Colombia en las que han participado la universidad EAFIT y la firma consultora INTEGRAL S.A, y después de considerar a través del método de Montecarlo las incertidumbres en los parámetros que definen las funciones de amplificación de los depósitos de suelo estudiados, se establece que para suelos tropicales como los considerados, dos valores razonables para las variables $n$ y $\varepsilon$ son 0.5 y 0.25 , respectivamente. Estos valores significan que para tener en cuenta la incertidumbre en las propiedades del suelo es suficiente con considerar la media de la función de amplificación más media desviación estándar, y que un valor razonable para esta última es igual a una cuarta parte del valor medio de la función de amplificación.

En definitiva, todo el problema de definición de los espectros de amenaza uniforme en la superficie del suelo queda en función del espectro de amenaza uniforme en el basamento rocoso, la media de la función de amplificación, $\overline{R R S}$, y los parámetros $n$ y $\varepsilon$.

La segunda simplificación que se incluye en el procedimiento propuesto es el hecho de que la función de amplificación, $R R S$, no depende exclusivamente de las propiedades del suelo, como si lo es la función de transferencia del suelo, $F T$, depende adicionalmente de la señal en el basamento rocoso. Esta dependencia es notable en periodos bajos, menores a $T_{r}$, y mucho menos importante en periodos altos (Roesset, 1970). La propuesta que se considera a continuación, incluye únicamente la caracterización de la función de amplificación para periodos superiores al fundamental del depósito de suelo, $T_{s}=1 / f_{s}$, que en la gran mayoría de los casos es superior a $T_{r}$, lo que razonablemente permite mantener la simplificación de definir la función de amplificación exclusivamente en términos de las propiedades del suelo.

Con base en un gran número de simulaciones, variando sistemáticamente las propiedades del suelo, $T_{s}$, $\xi_{s}$ e $I$, y las propiedades del espectro de amplitudes de Fourier en el basamento rocoso, $f_{c}, f_{m}, s, Q, \mu$ y $R$, y haciendo uso de la teoría de vibraciones aleatorias para calcular los espectros de respuesta a nivel de la roca y la superficie del suelo, se propone la siguiente función para representar la zona descendente de la función de amplificación: 


$$
\overline{R R S}=k+\left(R R S_{\max }-k\right)\left(\frac{T_{s}}{T}\right)^{\gamma} \quad\left(T>T_{s}\right)
$$

donde:

$R R S_{\max }: \quad$ Función de amplificación máxima que se presenta cuando $T=T_{s}$

$T_{s}$ : $\quad$ Periodo fundamental del depósito de suelo

$k$ : Parámetro

r._. Parámetro

Si se define un amortiguamiento equivalente, $\xi_{\text {eq }}$, (Roesset, 1970) como:

$$
\xi_{e q}=\xi_{s}+\frac{2.0}{\pi \cdot I}
$$

$R R S_{\max }, k$ y $\gamma$ se pueden estimar razonablemente bien en función de este amortiguamiento con las siguientes expresiones:

$$
\begin{aligned}
& R R S_{\text {max }}=\frac{1.0}{2 \xi_{\text {eq }}} \\
& k=1.0+\frac{1.0}{100 \xi_{\text {eq }}} \\
& \gamma=\frac{1.10}{\sqrt{\xi_{\text {eq }}}}
\end{aligned}
$$

Finalmente, y considerando que el espectro de respuesta en roca cae aproximadamente de manera inversa al periodo a partir de $T_{r}$ y hasta periodos hasta de $4.0 \mathrm{~s}$ para magnitudes de momento mayores a $6.5^{\circ}$, a continuación y con base en la función de amplificación propuesta en la ec. 15, se propone la forma de la rama descendente del espectro de respuesta de aceleraciones a nivel de la superficie del suelo.

$$
S a=\frac{\operatorname{Sar}_{\max } T_{r}}{T}\left[k+\left(R R S_{\text {max }}-k\right)\left(\frac{T_{s}}{T}\right)^{\gamma}\right]\left(1.0+n \sigma_{\varepsilon}\right) \quad\left(T_{s}<T<4.0 s\right)
$$

Esta forma funcional mantiene y combina adecuadamente las dos tendencias, la del espectro de respuesta a nivel del basamento rocoso y la de la función de amplificación 


\section{APLICACIÓN}

A continuación se aplica la propuesta descrita al caso de los espectros de diseño de la microzonificación sísmica de Medellín. Para esta ciudad, tanto los espectros de control de daños como de diseño están controlados por fuentes sísmicas que están a distancias que oscilan entre los 20 y los $100 \mathrm{~km}$ de distancia, lo que permite utilizar el modelo propuesto.

La microzonificación sísmica de Medellín se realizó entre 1996 y 1999 por parte de la empresa INTEGRAL S.A., la Facultad de Minas de la Universidad Nacional de Colombia, el INGEOMINAS y la Universidad EAFIT. Para esta se tuvieron en cuenta cerca de 1000 perforaciones, ensayos triaxiales cíclicos y ensayos de columna resonante sobre muestras inalteradas de suelo, ensayos geofísicos down hole en el sitio, y lo más importante, cerca de 600 registros acelerográficos de sismos de baja intensidad obtenidos por la Red acelerográfica de Medellín en 19 estaciones ubicadas a lo largo y ancho de la ciudad (Universidad EAFIT et al., 1999).

Para este caso se toma $T_{r}$ igual a $0.2 \mathrm{~s}, T_{s}$ igual al segundo periodo de quiebre del espectro, $T_{2}$, en vigencia, y se estima el amortiguamiento equivalente del suelo, $\xi_{\text {eq }}$, que reproduzca la aceleración espectral máxima a nivel de la superficie del suelo que se propone en los espectros de diseño actualmente vigentes.

A continuación se presentan los periodos fundamentales del suelo y los amortiguamientos equivalentes que resultan para cada una de las microzonas definidas en Medellín y para los dos espectros, de servicio y de diseño.

Tabla 2. Factores de amplificación, periodos fundamentales del suelo y amortiguamientos equivalentes del suelo para los espectros de servicio y diseño en las microzonas sísmicas de Medellín.

\begin{tabular}{|c|c|c|c|c|c|c|}
\hline ZONA & Fa Servicio & Fa Diseño & Ts Servicio & Ts Diseño & $\xi$ eq Servicio & $\xi$ eq Diseño \\
\hline 1 & 3.00 & 1.87 & 0.50 & 0.60 & 0.083 & 0.112 \\
\hline 2 & 2.99 & 2.13 & 0.30 & 0.40 & 0.140 & 0.147 \\
\hline 3 & 3.33 & 2.13 & 0.50 & 0.70 & 0.075 & 0.084 \\
\hline 4 & 2.40 & 1.33 & 0.60 & 0.65 & 0.087 & 0.145 \\
\hline 5 & 2.93 & 1.60 & 0.50 & 0.60 & 0.085 & 0.130 \\
\hline 6 & 1.87 & 1.33 & 0.40 & 0.50 & 0.167 & 0.188 \\
\hline 7 & 2.93 & 1.60 & 0.50 & 0.60 & 0.085 & 0.130 \\
\hline 8 & 2.40 & 1.47 & 0.65 & 0.75 & 0.080 & 0.114 \\
\hline 9 & 3.00 & 1.87 & 0.40 & 0.55 & 0.104 & 0.122 \\
\hline 10 & 3.33 & 2.13 & 0.40 & 0.50 & 0.094 & 0.118 \\
\hline 11 & 3.00 & 2.00 & 0.50 & 0.65 & 0.083 & 0.096 \\
\hline 12 & 3.33 & 2.13 & 0.65 & 0.70 & 0.058 & 0.084 \\
\hline 13 & 3.33 & 2.13 & 0.40 & 0.50 & 0.094 & 0.118 \\
\hline 14 & 1.87 & 1.60 & 0.50 & 0.55 & 0.134 & 0.142 \\
\hline
\end{tabular}


En la tabla 2 el factor de amplificación, $F a$, como se define en la microzonificación sísmica de Medellín, permite definir la aceleración espectral máxima a nivel de la superficie del suelo de acuerdo con la expresión:

$S a_{\max }=2.5 \mathrm{Fa} A_{a}$

Los amortiguamientos equivalentes calculados y presentados en la tabla 2 , tanto para servicio como para diseño, se aprecian muy bajos. Por ejemplo, para intensidades de diseño un amortiguamiento razonable del suelo es del orden de 0.07, y si a este se le suma el amortiguamiento por radiación de acuerdo con la ec. 16 y considerando una impedancia relativamente alta e igual a 7.0 , resulta un amortiguamiento equivalente igual a 0.16 , que es muy superior al promedio de los amortiguamientos equivalentes para diseño que se presentan en la tabla 2 e igual a 0.12 . Amortiguamientos tan bajos como el de las zonas 12 y 3 , iguales a 0.084 , para la misma impedancia propuesta, significaría amortiguamientos del suelo negativos. A la luz de la propuesta de este artículo, lo anterior significa que probablemente las aceleraciones espectrales máximas propuestas en la microzonificación sísmica de Medellín están sobrestimadas. No obstante lo anterior, en lo que sigue se mantienen los valores propuestos en la reglamentación vigente, y con base en estos y en la propuesta aquí presentada, se estima nuevamente la rama descendente de los espectros de control de daños y diseño.

Como la función de amplificación $R R S$ tiende a valores muy cercanos a 1.0 para periodos altos, la posible subestimación en los amortiguamientos equivalentes discutida en el párrafo anterior solo se reflejará en caídas más pronunciadas de los espectros, para finalmente, en periodos altos, reflejar los contenidos frecuenciales del movimiento en el basamento rocoso.

Con base en los valores de amortiguamiento equivalente calculados, en los periodos fundamentales del suelo propuestos en la microzonificación sísmica de Medellín, en un periodo de roca $T_{r}$ igual a $0.2 \mathrm{~s}$, y en los parámetros de incertidumbre del suelo, $n$ y $\varepsilon$ iguales a 0.5 y 0.25 , respectivamente, en las figs. 2 y 3 se muestran los espectros de control de daños y diseño para algunas de las microzonas sísmicas de Medellín.

Para tener más claridad sobre el significado de la propuesta presentada, se muestran también las gráficas de los espectros de aceleración originales de la microzonificación sísmica de Medellín y las gráficas de los espectros de aceleración corregidos de acuerdo a los acelerogramas registrados por la Red acelerográfica de Medellín, Jaramillo (2002). Igualmente, se muestran los espectros de respuesta de desplazamientos para todos los casos mencionados.

Es importante aclarar que en el caso de los espectros para control de daños se mantuvieron las expresiones aquí propuestas y que están basadas en que la frecuencia de esquina, $f_{c}$, es menor a $0.25 \mathrm{~Hz}$, es decir, que la caída definitiva del espectro de respuesta en roca está más allá de los 4.0s de periodo. Es posible que para los espectros de control de daños las magnitudes de momento sean menores a $6.5^{\circ}$ en cuyo caso, y de acuerdo a la tabla 1 , el periodo que marca la caída definitiva del espectro de aceleraciones en el basamento rocoso, $T_{c}$, es inferior a $4.0 \mathrm{~s}$, y en este caso, las expresiones propuestas y las gráficas presentadas solo serían válidas hasta este periodo. 

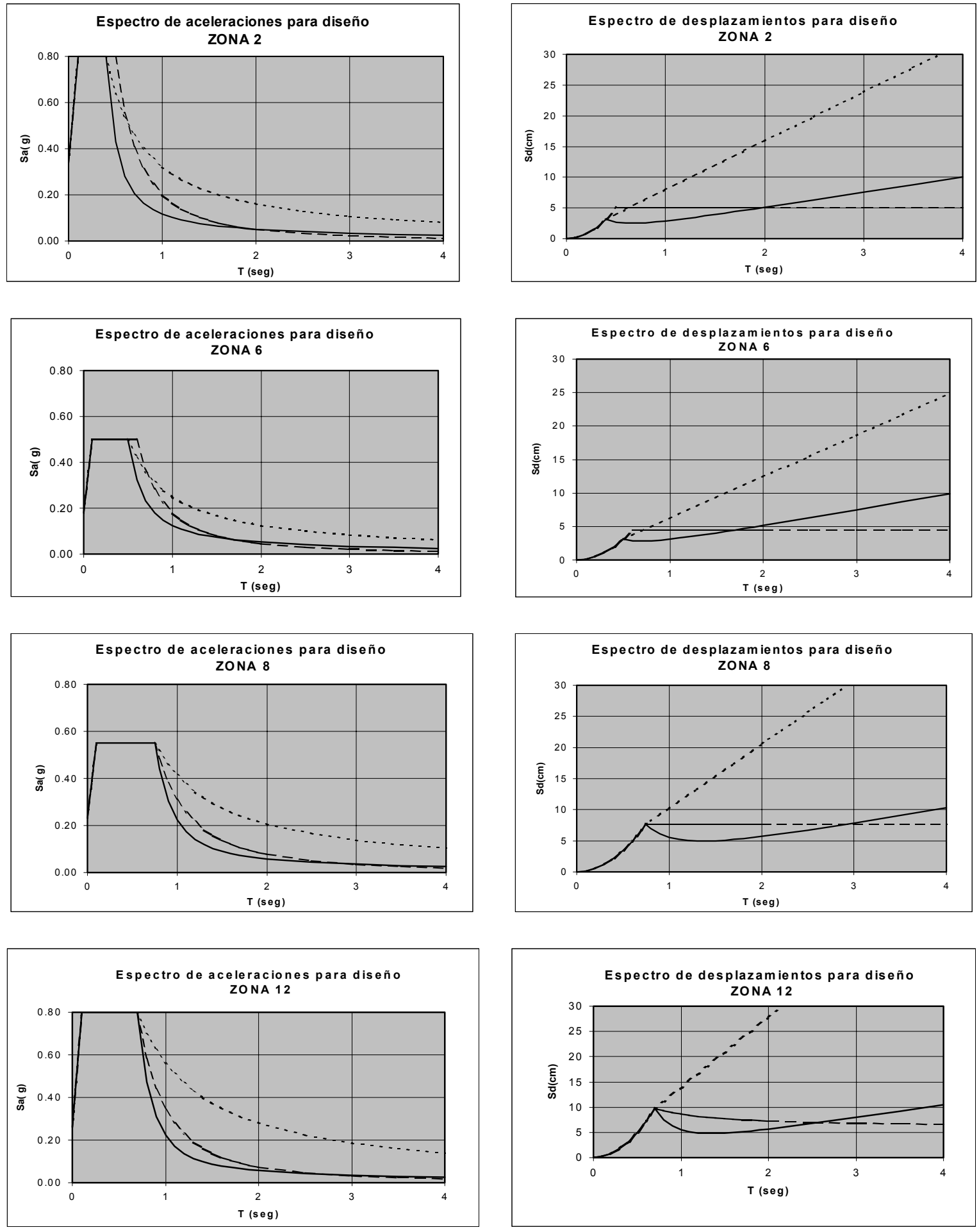

Figura 3. Espectros de aceleraciones y desplazamientos para diseño en algunas de las microzonas sísmicas de Medellín 

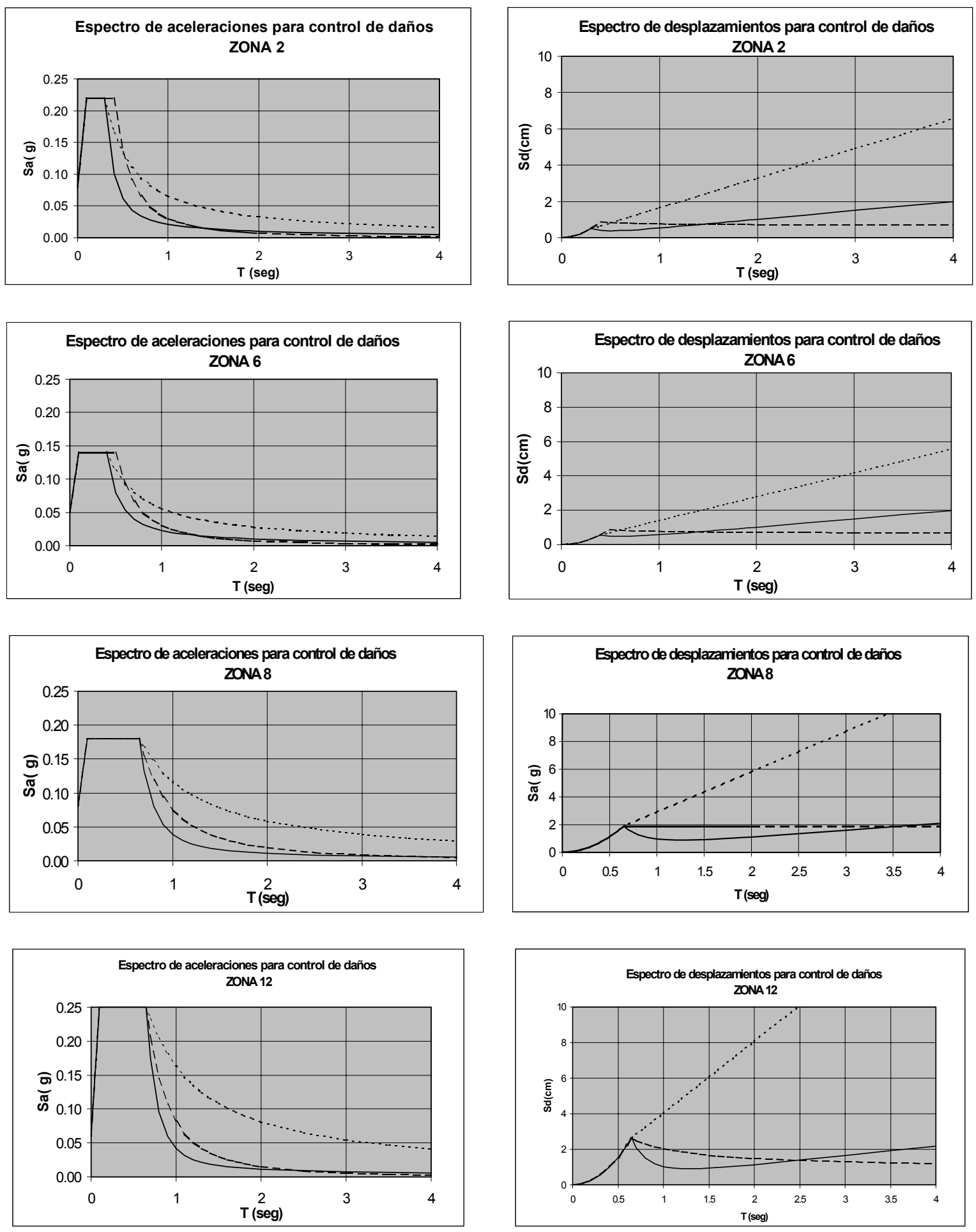

Figura 4. Espectros de aceleraciones y desplazamientos para control de daños en algunas de las microzonas sísmicas de Medellín 
A partir de $T_{c}$ el espectro de desplazamiento probablemente se mantenga constante e igual al desplazamiento máximo del suelo, indicando que el espectro de aceleraciones en roca a partir de este periodo cae inversamente proporcional al cuadrado del periodo. De acuerdo con la metodología aquí propuesta, esta última forma de caída del espectro en roca se multiplica por la función de amplificación, $R R S$, y se obtiene entonces el espectro de respuesta en la superficie del suelo para periodos mayores a $T_{c}$.

\section{ANÁLISIS DE RESULTADOS}

En términos de espectros de aceleración, las ramas descendentes de los espectros propuestos en Jaramillo (2002) y los propuestos en este trabajo, se asemejan bastante, presentándose algunas diferencias en que los aquí propuestos tienen caídas más pronunciadas al principio de la rama descendente y se suavizan un poco más al final, produciendo aceleraciones espectrales un poco mayores en periodos altos.

Nuevamente, se hace hincapié en que los espectros aquí propuestos y los propuestos en Jaramillo (2002) son marcadamente diferentes a los que actualmente propone la reglamentación.

En términos de espectros de desplazamiento, las diferencias entre los espectros propuestos por Jaramillo y los que propone la reglamentación son muy grandes, especialmente en periodos mayores a $2.0 \mathrm{~s}$.

Entre los espectros de desplazamiento propuestos en Jaramillo (2002) y los aquí propuestos hay una diferencia que más que numérica refleja un avance en la modelación del problema. En Jaramillo (2002) se propuso una expresión para definir directamente los espectros en la superficie del suelo, y ahora, se proponen expresiones independientes para modelar el espectro a nivel de roca y la función de amplificación del suelo, resultando en un modelo del espectro en la superficie del suelo que refleja los movimientos en el basamento rocoso y la amplificación producida por el depósito de suelo.

Los espectros de desplazamiento en la superficie del suelo muestran un pico en la frecuencia fundamental del suelo, después del cual caen un poco para volver a levantarse por efecto del contenido de bajas frecuencias del movimiento en el basamento rocoso. Son estas últimas ondas de baja frecuencia las responsables del levantamiento en periodos altos de algunos de los espectros de desplazamiento calculados en Jaramillo (2002).

Finalmente, se considera que algunos de los espectros de desplazamiento calculados en Jaramillo (2002) no presentan el levantamiento en periodos altos debido a las bajas magnitudes que los produjeron, es decir, que en estos movimientos de tan bajas intensidades el periodo de quiebre $T_{c}$ es menor a $2.0 \mathrm{~s}$, haciendo que desaparezca el efecto de levantamiento. 


\section{CONCLUSIONES}

Es claro que los espectros de aceleración en la superficie del suelo que actualmente proponen una gran cantidad de reglamentaciones sísmicas alrededor del mundo violan en la zona de periodos altos los principios de la dinámica estructural: los espectros de desplazamiento resultantes crecen indefinidamente, lo que resulta en un desplazamiento máximo del terreno infinito. Este hecho aunado a los límites que actualmente controlan el diseño sísmico de estructuras, como son los límites de derivas, resultan en sobre diseños que superan en la mayoría de los casos cualquier límite racional que se pueda fijar a un factor de seguridad.

En este trabajo, basados en la forma propuesta por el modelo $w^{2}$ para el espectro de amplitudes de Fourier a nivel del basamento rocoso, y la función de transferencia del suelo, y a partir de estos y la teoría de vibraciones aleatorias, $T V A$, se proponen formas para los espectros de respuesta de aceleraciones a nivel del basamento rocoso, funciones de amplificación de espectros de respuesta, y finalmente, espectros de respuesta de aceleraciones a nivel de la superficie del suelo.

Las formas propuestas en este trabajo permiten, a partir de información como la aceleración máxima para diseño en el basamento rocoso, $A_{a}$, el periodo de amplificación máxima del espectro de aceleraciones en roca, $T_{r}$, y algunos datos del depósito de suelo, como su periodo fundamental, $T_{s}$, el amortiguamiento del suelo, $\xi_{s}$, y la impedancia de este con el basamento rocoso, $I$, definir la rama descendente de los espectros de respuesta a nivel de la superficie del suelo.

Las formas resultantes aplicadas a las ramas descendentes de los espectros de respuesta definidos en la microzonificación sísmica de Medellín, permiten explicar algunos efectos visualizados pero no explicados completamente en trabajos anteriores basados en el procesamiento de acelerogramas registrados.

\section{AGRADECIMIENTOS}

El autor agradece la colaboración del ingeniero Andrés Felipe Vélez en la programación de las expresiones de la Teoría de Vibraciones Aleatorias, TVA, base de las simulaciones que permitieron estimar las expresiones propuestas. Igualmente, el autor agradece los comentarios y sugerencias de dos revisores anónimos del manuscrito, que sin lugar a dudas mejoraron sustancialmente este trabajo. Finalmente, el autor agradece el patrocinio de la Universidad EAFIT para poder llevar a cabo este estudio.

\section{REFERENCIAS}

Academia de Ciencias Físicas Matemáticas y Naturales (1997), Diseño sismorresistente, especificaciones y criterios empleados en Venezuela, Caracas, Coordinador José Grases. 
AIS (1984), "Código colombiano de construcciones sismo-resistentes, decreto 1400 de junio 7 de 1984”, Asociación Colombiana de Ingeniería Sísmica (AIS), Santafé de Bogotá.

AIS (1999), "Normas colombianas de diseño y construcción sismo-resistente, Ley 400 de 1997, decreto 33 de 1998”, Asociación Colombiana de Ingeniería Sísmica (AIS), Santafé de Bogotá.

ASIA (1997), "Normas técnicas: para diseño por sismo, para diseño por viento, y sus comentarios", Asociación Salvadoreña de Ingenieros y Arquitectos (ASIA) y Ministerios de Obras Públicas de la República del Salvador, El Salvador.

Avilés, J, H R Aguilar y L E Pérez-Rocha (1995), “Interacción dinámica suelo-estructura en el valle de México”, Revista de Ingeniería Sísmica, SMIS, No. 50, pp. 31-45.

Boore, D M (1983), "Stochastic simulation of high-frequency ground motions based of seismological models of the radiated spectra", Bulletin of Seismological Society of America, No. 73, pp. 1865-1894.

Boore, D M y W B Joyner (1984), “ A note on the use of random vibration theory to predict peak amplitude of transient signals, Bulletin of Seismological Society of America, No. 74, pp. 20352039.

Brune, J N (1970), "Tectonic stress and the spectra of seismic shear waves from earthquakes", Journal of Geophysical Research, No. 75, pp. 4997-5009.

Cartwright, D E y M S Longuett_Higgins (1956) "The statistical distribution of the maxima of a random function", Memorias, Royal Society of London, Vol. A237, pp. 212-232.

Chopra, A K (2001), Dynamics of Structures, Theory and applications to earthquake engineering, segunda edición, Prentice Hall.

Dobry, R, R D Borcherdt, C B Crouse, I M Idriss, W B Joyner, G R Martin, M S Power, E E Rinne y R B Seed (2000), "New site coefficients and site classification system used in recent building seismic code provisions, Earthquake Spectra, Vol. 16, No. 1, pp. 41-67.

Hanks, T H y H Kanamori (1979), “A moment magnitude scale", Journal of Geophysical Research, No. 84, pp. 2348-2350.

Hermann, R B (1985), "An extension of random vibration theory estimates of strong ground motion to large distances", Bulletin of Seismological Society of America, No. 73, pp. 157-171.

Jaramillo, J D (2002), "Espectros de diseño sísmico para la ciudad de Medellín”, Revista Internacional de Ingeniería de Estructuras, Vol. 7, No. 1, pp. 1-19.

Leyendecker, E V, R J Hunt, A D Franklen y K S Rukstales (2000), “Development of maximum considered earthquake ground motion maps", Earthquake Spectra, Vol. 16, No. 1, pp. 21-40.

Newmark, N M y E Rosenblueth (1971), Fundamentals of earthquake engineering, Prentice Hall.

NTCS-2001 (2001), "Propuesta de normas técnicas complementarias para diseño por sismo", http://www.smie.org.mx/articulos , abril.

Ordaz, M, E Miranda y J Avilés (2000), “Propuesta de espectros de diseño por sismo para el DF”, Inédito. 
Roesset, J M (1969), "Fundamentals of soil amplification", Massachusetts Institute of Technology.

Singh, S K, M Ordaz, J G Anderson, M Rodriguez, R Quaas, E Mena, M Ottaviani y D Almora (1989), "Analysis of near-source strong motion recordings along the Mexican subduction zone", Bulletin of Seismological Society of America, No. 79, pp. 1697-1717.

Universidad EAFIT, Integral S.A, Ingeominas y Facultad Nacional de Minas (1999), "Instrumentación y microzonificación sísmica del área urbana de Medellín", Publicación del Municipio de Medellin, Secretaría privada, 135p. 